\title{
Colon Polyp Observed During
}

\section{Examination}

National Cancer Institute

\section{Source}

National Cancer Institute. Colon Polyp Observed During Examination. NCI Thesaurus.

Code C162529.

Direct observation of a colon polyp upon examination, during surgical procedure, or colonoscopy. 